\title{
Analyzing AbrB-Knockout Effects through Genome and Transcriptome Sequencing of Bacillus licheniformis DW2
}

\author{
Cheng-Cheng Shu ${ }^{1}$, Dong Wang ${ }^{2}$, Jing Guo ${ }^{1}$, Jia-Ming Song ${ }^{1}$, Shou-Wen Chen ${ }^{2}$, \\ Ling-Ling Chen ${ }^{1}$ and Jun-Xiang Gao ${ }^{1 *}$
}

'Agricultural Bioinformatics Key Laboratory of Hubei Province, College of Informatics, Huazhong Agricultural University, Wuhan, China, ${ }^{2}$ State Key Laboratory of Agricultural Microbiology, College of Life Science and Technology, Huazhong Agricultural University, Wuhan, China

As an industrial bacterium, Bacillus licheniformis DW2 produces bacitracin which is an important antibiotic for many pathogenic microorganisms. Our previous study showed AbrB-knockout could significantly increase the production of bacitracin. Accordingly, it was meaningful to understand its genome features, expression differences between wild and AbrB-knockout ( $\triangle \mathrm{AbrB}$ ) strains, and the regulation of bacitracin biosynthesis. Here,

OPEN ACCESS

Edited by:

Feng Gao,

Tianjin University, China

Reviewed by:

Mingfeng Cao,

lowa State University, United States

Ozlem Ates Duru,

Nişantaşı University, Turkey

${ }^{*}$ Correspondence:

Jun-Xiang Gao

gao200@mail.hzau.edu.cn

Specialty section:

This article was submitted to

Evolutionary and Genomic

Microbiology,

a section of the journal

Frontiers in Microbiology

Received: 30 November 2017 Accepted: 09 February 2018

Published: 26 February 2018

Citation:

Shu C-C, Wang D, Guo J,

Song J-M, Chen S-W, Chen L-L and

Gao J-X (2018) Analyzing

AbrB-Knockout Effects through

Genome and Transcriptome

Sequencing of Bacillus licheniformis

DW2. Front. Microbiol. 9:307.

doi: 10.3389/fmicb.2018.00307 we sequenced, de novo assembled and annotated its genome, and also sequenced the transcriptomes in three growth phases. The genome of DW2 contained a DNA molecule of 4,468,952 bp with $45.93 \%$ GC content and 4,717 protein coding genes. The transcriptome reads were mapped to the assembled genome, and obtained 4,102 4,536 expressed genes from different samples. We investigated transcription changes in $B$. licheniformis DW2 and showed that $\triangle$ AbrB caused hundreds of genes up-regulation and down-regulation in different growth phases. We identified a complete bacitracin synthetase gene cluster, including the location and length of bacABC, $b c r A B C$, and $b a c T$, as well as their arrangement. The gene cluster bcrABC were significantly up-regulated in $\triangle \mathrm{AbrB}$ strain, which supported the hypothesis in previous study of bcrABC transporting bacitracin out of the cell to avoid self-intoxication, and was consistent with the previous experimental result that $\triangle \mathrm{AbrB}$ could yield more bacitracin. This study provided a high quality reference genome for $B$. licheniformis DW2, and the transcriptome data depicted global alterations across two strains and three phases offered an understanding of AbrB regulation and bacitracin biosynthesis through gene expression.

Keywords: Bacillus licheniformis, reference genome, transcriptome, AbrB-knockout, differentially expressed genes, bacitracin biosynthesis

\section{INTRODUCTION}

Bacillus licheniformis (B. licheniformis) is a Gram-positive bacterium that is widely used in multiple fields: in agriculture as a probiotic and microbial fertilizer (Pötter et al., 2001), and in biotechnology industry for the production of enzymes, acetoin and poly- $\gamma$-glutamic acid ( $\gamma$-PGA) (Veith et al., 2004; Liu et al., 2011; Konglom et al., 2012). This facultative anaerobic organism can 
also produce a variety of antibiotics such as bacitracin, which is an important peptide antibiotic for many pathogenic microorganisms secreted by certain strains of $B$. licheniformis and B. subtilis (Johnson et al., 1945; Mcinerney et al., 1990). The branched cyclic dodecyl peptide bacitracin is the most active against other Gram-positive and certain Gramnegative microorganisms, which is synthesized by a large nonribosomal multi-enzyme complex bacABC (Konz et al., 1997). The AbrB gene of $B$. licheniformis and B. subtilis is known as an important global transcription repressor, but also act as an activator for some genes by binding to promoters (Kim et al., 2003). AbrB has a wide range of regulatory functions, including cell wall and membrane synthesis, bacterial chemotaxis, antibiotic synthesis, amino acid synthesis and transport, protein modification and so on (Qian et al., 2002; Strauch et al., 2007). AbrB directly regulates more than 100 genes and indirectly influence hundreds genes. The evidence suggests that expression level of $\mathrm{AbrB}$ is inhibited by another regulatory gene Spo0A-P, leading to an opposite tendency of AbrB gene expression compares with that of Spo0A-P, i.e., high expression during the logarithmic phase and low expression during stationary phase (Strauch et al., 1990; Shafikhani and Leighton, 2004; Banse et al., 2008; Chumsakul et al., 2011).

Since the project of $B$. subtilis genome was completed as the first gram-positive bacterium (Kunst et al., 1997), more and more Bacillus strains have been sequenced including B. licheniformis ATCC14580, 9945A, WX-02 and DSM13; B. subtilis CGMCC 12426, BSD-2, and so on (Veith et al., 2004; Guo et al., 2015; Li et al., 2015; Liu et al., 2016). In contrast, some other studies are limited to single or several proteins and metabolites, for example, the production of increased isopentenyl pyrophosphate (IPP) (Cain et al., 1993; Chalker et al., 2000), the secretion of exopolysaccharides (Pollock et al., 1994) and bcrABC protein transporting bacitracin in B. subtilis (Podlesek et al., 1995, 2000). Although these studies provide great advances, there has never been a comprehensive research focused on the effects of AbrBknockout in term of whole genome and transcriptome, especially the effect on the bacitracin gene cluster.

Our previous study showed that AbrB-knockout could significantly increase the production of bacitracin, and the yields were $17.5 \%$ higher than that of the wild-type strain (Wang et al., 2017). As an industrial B. licheniformis strain, DW2 can produce an extra yield using the improvement way of AbrBknockout in industrial application. In the present study, we sequenced, annotated the genome and transcriptome of an important industrial B. licheniformis strain DW2, and obtained the following results. (1) The genome of $B$. licheniformis DW2 is $4,468,952$ bp with $45.93 \%$ GC content and 4,717 predicted coding sequences (CDSs). (2) B. licheniformis DW2 has good collinearity with other $B$. licheniformis strains, and a unique $\sim 100 \mathrm{~kb}$ genomic sequences. (3) N-6-methylated adenines (6mA) and 4methylated cytosines $(4 \mathrm{mC})$ methylation of $B$. licheniformis DW2 was analyzed, and two $6 \mathrm{~mA}$ motifs were identified. (4) There are $369 \sim 1,517$ differentially expressed genes (DEGs) between the transcriptome of wild and $\triangle \mathrm{AbrB}$ strains in logarithmic growth phase, transitional phase and stationary growth phase.
(5) We identified the location and length of bacitracin synthetase gene cluster in $B$. licheniformis DW2. In this gene cluster, the transporter genes $b c r A B C$ were significantly highly expressed in $\triangle \mathrm{AbrB}$ strain, which supported the assumption that $\mathrm{bcr} A B C$ genes could pump bacitracin out to avoid self-intoxication. The study produced a reference genome of $B$. licheniformis, also enabled us to highlight global changes in gene expression and provided theory and data support for industrial process of bacitracin production.

\section{MATERIALS AND METHODS}

\section{Genomic DNA Preparation}

The DW2 strain used in this study was provided by China Center for Type Culture Collection (CCTCC), whose number was CCTCC M2011344. B. licheniformis DW2 was grown on Luria Bertani (LB) agar plates or in LB broth at $37^{\circ} \mathrm{C}$, supplemented with antibiotics (tetracycline, $20 \mathrm{mg} / \mathrm{mL}$; ampicillin, $50 \mathrm{mg} / \mathrm{mL}$; kanamycin, $20 \mathrm{mg} / \mathrm{mL}$ ) when necessary. The seed culture $(1 \mathrm{~mL})$ was inoculated into a $250 \mathrm{~mL}$ Erlenmeyer flask containing $20 \mathrm{~mL}$ bacitracin fermentation medium [6\% soybean meal, $4.5 \%$ starch, $\left.0.1 \%\left(\mathrm{NH}_{4}\right)_{2} \mathrm{SO}_{4}, 0.6 \% \mathrm{CaCO}_{3}, \mathrm{pH} \quad 6.5 \sim 7.0\right]$, followed by $40 \mathrm{~h}$ incubation on a rotary shaker (180 rpm) at $37^{\circ} \mathrm{C}$. Genomic DNA was extracted using a standard SDSPhenol procedure (Sambrook et al., 1982) according to the manufacturer's instructions, combined with phenol-chloroform extraction and RNase A treatment.

\section{PacBio SMRT Sequencing}

We determined the complete genome sequence of $B$. licheniformis DW2 using PacBio single molecule real-time (SMRT) technology. We prepared a 3 to $20 \mathrm{~kb}$ genomic DNA library suitable for P6/C4 chemistry. Using two SMRT cell on PacBio RSII sequencing platform, 116,486 polymerase reads with mean read length of 4,268 bp were obtained. The polymerase reads were assembled de novo with Hierarchical Genome Assembly Process 3 (HGAP3) within the SMRT Analysis version 2.3.0 software (Chin et al., 2013). Subsequently, the best assembly was selected, and Minimus 2 was used for trimming the circular contig (Sommer et al., 2007). The replication origin was determined by aligning the DW2 genomic sequence with $B$. licheniformis DSM13.

For identification of methylated bases and modification motifs, the "RS_Modification_and_Motif_Analysis.1" protocol in SMRT Portal under default parameter settings was used, with the assembled genome. Putative restriction modification systems have been identified using the Restriction-ModificationFinder1.0 server $^{1}$ based on the Restriction Enzyme database (REBASE) (Roberts et al., 2015).

\section{The Annotation of $B$. licheniformis DW2 Genome}

After the complete sequence was obtained, the genome was annotated using the rapid annotation tool RAST (Ogata et al., 1999; Aziz et al., 2008). In addition, the unigenes

\footnotetext{
${ }^{1}$ https://cge.cbs.dtu.dk/services/Restriction-ModificationFinder-1.0/
} 
were annotated by the public protein databases of Kyoto Encyclopedia of Genes and Genomes (KEGG) (Galperin et al., 2015), Cluster of Orthologous Groups of Proteins (COG) (Gene Ontology Consortium, 2015), Swiss-Prot protein ${ }^{2}$, NCBI nonredundant $\left(\mathrm{NR}^{3}\right)$, and Gene Ontology (GO) (Lagesen et al., 2007) using BLASTP to get corresponding functional annotation information. As each sequence had many alignments, we retain the optimal comparison results as the annotation of the gene to ensure its accuracy. The predictions of rRNA and tRNA were performed using RNAmmer (Lowe and Eddy, 1997) and tRNAscan-SE (Zhang et al., 2000), respectively. Insertion sequence (IS) elements were predicted with IS Finder (Benson, 1999).

\section{Whole-Genome Alignment and Identification of Operon Structures}

We constructed and visualized the multiple genome alignment of four complete genomes of B. licheniformis strains DW2, ATCC14580, 9945A and WX-02 using Mauve v2.4.0 (Darling et al., 2010). Pairwise collinear comparisons of the four genome sequences were performed using Mummer3 (Kurtz et al., 2004). Operon of $B$. licheniformis DW2 was identified using DOOR 2.0 algorithm for prokaryotic operon analysis (Mao et al., 2014).

\section{RNA Isolation and Library Preparation for ssRNA-seq}

RNA samples were isolated at three time points for two bacterial samples (wild strain and $\triangle \mathrm{AbrB}$ strain): $14 \mathrm{~h}$ (logarithmic growth phase), $22 \mathrm{~h}$ (transitional phase) and $25 \mathrm{~h}$ (stationary phase). Cells were collected by centrifugation at $12,000 \mathrm{rpm}$ for $5 \mathrm{~min}$, and then transferred to a $10-\mathrm{ml}$ centrifuge tube after grinding in liquid nitrogen. Cells were then lysed in $1 \mathrm{~mL}$ of TRIzol for 30-60 s. To the lysate, $200 \mu \mathrm{L}$ of chloroform was added, and the sample was then mixed by inversion and incubated at room temperature for $15 \mathrm{~min}$. The sample was then centrifuged at $12,000 \mathrm{rpm}$ for $15 \mathrm{~min}$ at $4^{\circ} \mathrm{C}$, and supernatant was precipitated with an equal volume of isopropanol at room temperature for $10 \mathrm{~min}$. After centrifugation, the supernatant was discarded, and the pellet was air dried and dissolved in 20-40 $\mu \mathrm{L}$ of RNase-free water. Total RNA was treated with RNase-free DNase I for $30 \mathrm{~min}$ at $37^{\circ} \mathrm{C}$ to remove genomic DNA. RNA concentration was measured using Qubit ${ }^{\circledR}$ RNA Assay Kit in Qubit $^{\circledR} 2.0$ Fluorometer (Life Technologies, Carlsbad, CA, United States). At the same time, RNA integrity was assessed using the RNA Nano 6000 Assay Kit of the Bioanalyzer 2100 system (Agilent Technologies, Santa Clara, CA, United States). Each sample had three replicates, and all biological replicates were processed in separate batches from each other.

Libraries for ssRNA sequencing (Novogene Experimental Department) were constructed at three time points $(14,22$, and $25 \mathrm{~h}$ ). A total amount of $3 \mu \mathrm{g}$ RNA per sample was used as

${ }^{2} \mathrm{http}: / /$ www.expasy.ch/sprot

${ }^{3}$ http://www.ncbi.nlm.nih.gov input material for the RNA sample preparations. Sequencing libraries were generated using NEBNext ${ }^{\circledR}$ Ultra $^{\mathrm{TM}}$ Directional RNA Library Prep Kit for Illumina ${ }^{\circledR}$ (NEB, United States) following manufacturer's recommendation and index codes were added to attribute sequences to each sample. Briefly, (epicentre Ribo-Zero ${ }^{\mathrm{TM}}$ ) fragmentation was carried out using divalent cations under elevated temperature in NEBNext First Strand Synthesis Reaction Buffer (5X). First strand cDNA was synthesized using random hexamer primer and M-MuLV Reverse Transcriptase (RNaseH-). Second strand cDNA synthesis was subsequently performed using DNA Polymerase I and RNase $H$. In the reaction buffer, dNTPs with dTTP were replaced by dUTP. Remaining overhangs were converted into blunt ends via exonuclease/polymerase activities. After adenylation of $3^{\prime}$ ends of DNA fragments, NEBNext Adaptor with hairpin loop structure were ligated to prepare for hybridization. In order to select cDNA fragments of preferentially $150 \sim 200$ bp in length, the library fragments were purified with AMPure XP system (Beckman Coulter, Beverly, MA, United States). Then $3 \mu$ l USER Enzyme (NEB, United States) was used with size-selected, adaptor-ligated CDNA at $37^{\circ} \mathrm{C}$ for $15 \mathrm{~min}$ followed by $5 \mathrm{~min}$ at $95^{\circ} \mathrm{C}$ before PCR. Then PCR was performed with Phusion High-Fidelity DNA polymerase, Universal PCR primers and Index (X) Primer. At last, products were purified (AMPure XP system) and library quality was assessed on the Agilent Bioanalyzer 2100 system.

\section{Mapping Reads to B. licheniformis DW2 Genome and Analyzing Differentially Expressed Genes}

All the raw reads were initially processed to obtain clean reads by the following steps: (1) reads with adaptor were discarded; (2) reads with ambiguous bases (undetermined bases, $N$ ) larger than $10 \%$ were removed; (3) low-quality reads that contained more than $50 \% Q$-value $<5$ bases were discarded. After filtering, all clean reads were aligned to the assembled genome using Bowtie2 (Langmead and Salzberg, 2012), and reads with ambiguous alignments or more than three mismatches were discarded. Only uniquely mapped reads were used for further analysis. The perbase-format coverage depth and read counts were calculated using BEDTools (Quinlan and Hall, 2010). Because different samples may have different total read counts, sequencing depth, and biases, the normalized transcription level of genes was expressed in reads per kilobase of ORF per million mapped reads (RPKM) (Mortazavi et al., 2008). The transcriptome raw reads have been deposited in NCBI Sequence Read Archive database under accession number SRP045205. In addition, we generated a distance matrix for expression data based on RPKM values by using Pearson correlation as dissimilarity metric (El-Sharnouby et al., 2013). Clustering was performed and deprograms were generated using the cluster package in $\mathrm{R}$.

Differentially expressed genes between wild strain and $\triangle \mathrm{AbrB}$ strain were identified using the DESeq R package with the MARS (MA-plot-based method with Random Sampling) model (Anders and Huber, 2010). The DEGs were screened with the false discovery rate (FDR) threshold of $\leq 0.01$ and an absolute value of fold change $(\mathrm{FC}) \geq 2$. 


\section{RESULTS AND DISCUSSION}

\section{Genomic Features and Comparative Genomics of $B$. licheniformis DW2}

Bacillus licheniformis DW2 genome has one circular DNA with $4,468,952$ bp length and $45.93 \%$ GC content. The DNA molecule (Figure 1) contained 4,717 predicted CDSs accounting for $\sim 87 \%$ of the genome. Among the predicted CDSs, 3,896 were assigned biological functions or putative functions and 821 could be annotated as hypothetical protein. In addition, 24 rRNA genes and 81 tRNA-coding genes were identified, and 71 IS elements were found. Additional information about the genome statistics is shown in Table $\mathbf{1 .}$

To obtain further insights into B. licheniformis DW2 genome, we performed comparative genomics analysis with other three available B. licheniformis genomes: DW2, ATCC14580, 9945A and WX-02 (Table 2). B. licheniformis DW2 had the largest genome size and number of predicted CDSs, IS and tandem repeat sequence. However, the number of rRNAs and tRNAs was consistent with the others and the number of rRNAs was almost same. The multi-genome alignments showed the four genomes shared homologous blocks in order (Figure 2A). We found that $B$. licheniformis DW2 genome contained a specific fragment at $3.3 \sim 3.4 \mathrm{Mb}$ with $113 \mathrm{CDSs}$, most of them were hypothetical proteins and phage-associated proteins (such as phage-like protein, phage protein, phage capsid, and scaffold). Considering that only B. licheniformis DW2 can highly yield peptide antibiotics in the above four strains, we hypothesized that this region might be associated with the yield of peptide antibiotics and inhibition of pathogens. The B. licheniformis DW2 genome was collinear with the other three B. licheniformis strains (Supplementary Figure S1), and it has the best consistency with B. licheniformis 9945A.

\section{DNA Methylation Features in $B$. licheniformis DW2}

DNA methylation is an essential epigenetic modification that can change the activity of a DNA segment without changing the sequence. It typically represses gene transcription if it locates in a gene promoter (Laird, 2010). In bacteria DNA methylation often acts as a cellular defense system against phage infection that confers a selective advantage to the host bacterium (Sitaraman, 2016). If a foreign DNA is introduced into the cell, it will be degraded by sequence-specific restriction enzymes while the methylated DNA of the bacteria is not recognized by the restriction enzymes. Therefore, DNA methylation can be viewed as a primitive immune system to protect bacteria from bacteriophage attack (Sitaraman, 2016). DNA methylation transfers methyl groups from adenosylmethionine to adenine or cytosine by DNA methyltransferase to form 6methyladenine (6mA), 4-methyl cell (4-Methylcytosine, 4mC) and 5-Methylcytosine $(5 \mathrm{mC})$, of which $6 \mathrm{~mA}$ and $4 \mathrm{mC}$ are mainly found in prokaryotes (Kumar et al., 1994; Sánchezromero et al., 2015). PacBio SMRT technology has been used to simultaneously detect DNA methylation modification during single-molecule genome sequencing (Flusberg et al., 2010). In order to characterize the methylation of B. licheniformis DW2, using the SMRT Analysis Modification and Motif detection, we identified 2,939 $6 \mathrm{~mA}, 2,7844 \mathrm{mC}$ and 88,319 unspecific "modified bases" where the type of modification was not recognized by the software.

We then detected candidate methylation motifs using a sliding window of $5 \mathrm{~kb}$, and identified two dominant motifs (GCANNNNNNNNRTRTC and GAYAYNNNNNNNNTGC), both of them were recognized by N-6 adenine-specific methyltransferases. Figure $\mathbf{2 B}$ shows the distribution of two motifs in $B$. licheniformis DW2 genome, approximately $40 \%$ of $6 \mathrm{~mA}$ bases are clustered into the two motifs. However, there is no consensus motif for $4 \mathrm{mC}$-methylated bases or other unspecific modified bases. We aligned the two motifs to a comprehensive restriction-modification (RM) system database REBASE (Roberts et al., 2015) to make sure whether the two motifs have been identified in other species, and found that both motifs could not match existing recognition sequences of the restriction systems.

\section{Prediction and Analysis of Operons in B. licheniformis DW2}

We predicted 1021 operons based on RNA-seq data of B. licheniformis DW2 using software DOOR. Among these operons, $446(43.7 \%)$ of them are composed of two genes and 19 of them are composed of more than 10 genes. Figure 2C shows the operon acoABCL associated with acetoin catabolism, where $a c o R$ is a transcriptional regulator, $a c o L$ is dihydrolipoamide dehydrogenase, and $a \operatorname{co} A B C$ is a gene related to acetoin dehydrogenase (Ali et al., 2001). Many microorganisms can convert carbohydrates into acetone during glycolysis to avoid over-acidification. As shown in the figure, these genes had high expression at 22 and $25 \mathrm{~h}$ in DW2 strain, while they were almost not expressed at $14 \mathrm{~h}$ in DW2 and all the three time points in $\triangle \mathrm{AbrB}$ strain. These results indicate that AbrB may have a great effect on the expression of this operon, and this effect is time-specific, which has a significant effect in stationary phase and no obvious effect in logarithmic phase.

Figure $2 \mathrm{D}$ shows the stage $\mathrm{V}$ sporulation protein. The spoVA operon of B. subtilis is expressed only in the developing spore during sporulation and at least five SpoVA proteins, SpoVAA, $B,-C,-D$, and $-E b$, are necessary for normal $B$. subtilis spore formation (Perez-Valdespino et al., 2014). Many genes of $\Delta \mathrm{AbrB}$ strain showed much higher expression than DW2 strain, which fitted the basic fact that $\mathrm{AbrB}$ was a global regulatory gene and had inhibition effect on many genes. The expression level significantly increased when AbrB gene was knocked out. The $\triangle \mathrm{AbrB}$ genes at $14 \mathrm{~h}$ were highly expressed compared with that at 22 and $25 \mathrm{~h}$. In general, B. licheniformis would not sporulate during logarithmic growth phase. As for spoVAC and spoVAB, we observed that the expression at 22 and $25 \mathrm{~h}$ were significantly lower than those at $14 \mathrm{~h}$, which indicated they were activated during logarithmic growth phase. These results suggest that knockout of AbrB is favorable for sporulation. Then the knockout of AbrB may be able to directly relieve the inhibition of $\mathrm{AbrB}$ on other genes related to spore synthesis, thereby increasing 


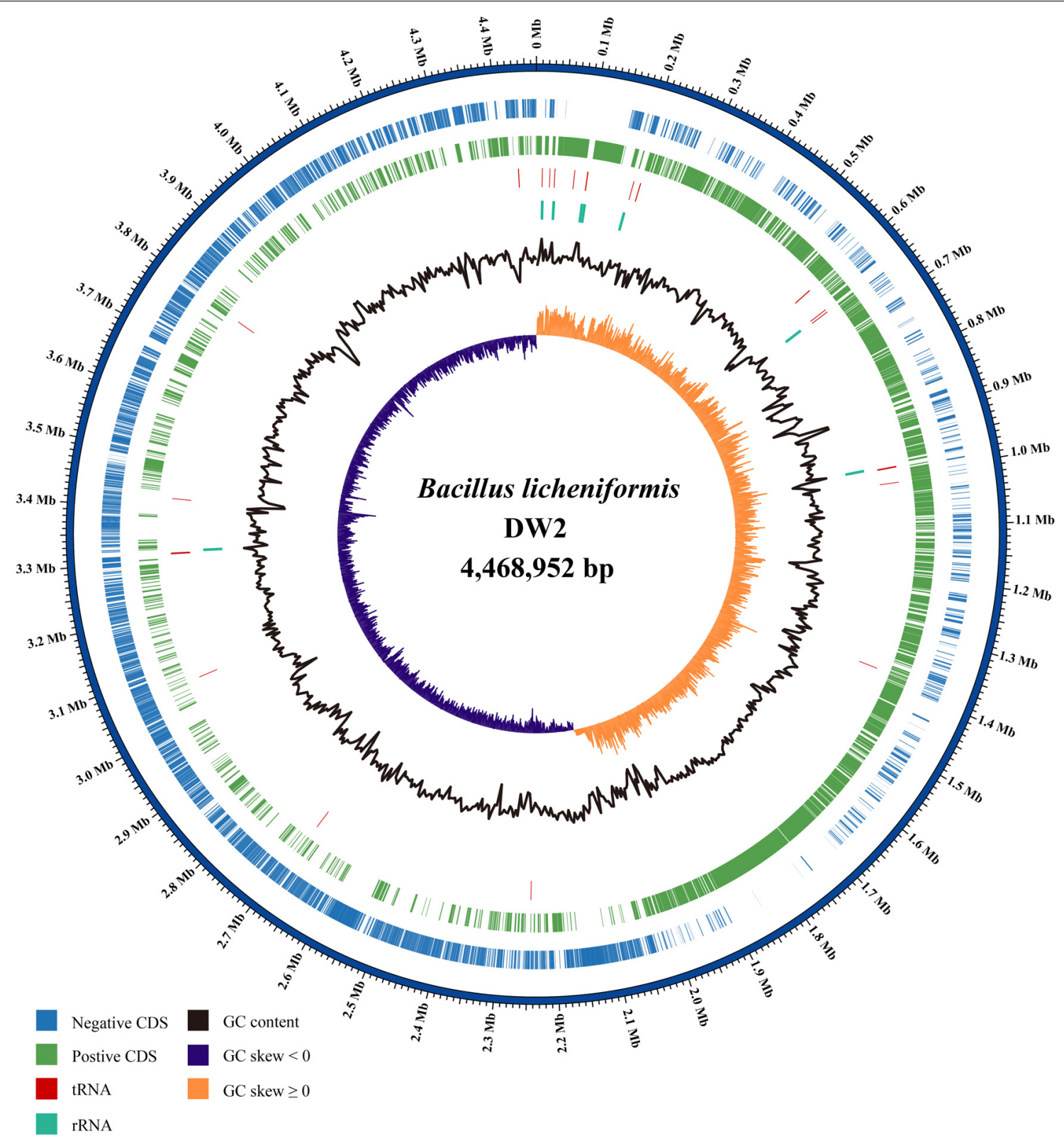

FIGURE 1 | The circular plot of $B$. licheniformis DW2 chromosome. Circles are numbered from 1 (outermost) to 7 (innermost). Circle 1 represents the whole chromosome; Circles 2 and 3 show the locations of predicted CDSs on the positive and negative strands, respectively; Circle 4, tRNA genes; Circle 5, rRNA genes; Circle 6, \%G + C; Circle 7, GC skew $((G-C) /(G+C))$.

the transcription level of spore-related genes and promote the synthesis of spores.

\section{Differentially Expressed Genes (DEGs) of Wild and $\triangle \mathrm{AbrB} B$. licheniformis DW2 Strains under Different Conditions}

In the experiment of RNA isolation and preparation, we performed three biological replicates. Consequently, we obtained RNA-seq data at three time points from two strains and three biological replicates, 18 sets of RNA-seq data in total. It is important to examine the correlation of the three biological replicates. We calculated the Spearman coefficient of correlation among the data sets. The results in Figure 3A show that the RPKM of biological replicates are highly correlated. The AbrB gene has been completely knocked out according to the expression levels (Figure 3B).

We screened the expressed genes of wild-type strain and $\triangle \mathrm{AbrB}$ strain at different time points with the threshold of $\mathrm{RPKM}>1$, and the number of expressed genes in different time points were in the range of 4,102 4,536 (Figure 3C). 
DEGs were obtained using DEseq package between the two data sets of both strains and three growth phases (Table 3 ). As shown in Figure 3D, the number of expressed genes of

TABLE 1 | Genome statistics of B. licheniformis DW2.

\begin{tabular}{lc}
\hline Feature & Value \\
\hline Genome size (bp) & $4,468,952$ \\
DNA coding (bp) & $3,918,654$ \\
DNA G+C (bp) & $2,052,745$ \\
DNA scaffolds & 1 \\
Total genes & 4,822 \\
Protein coding genes & 4,717 \\
RNA genes & 105 \\
Genes with function predictions & 3,896 \\
Genes assigned to COGs & 3,205
\end{tabular}

$\Delta \mathrm{AbrB}$ increased significantly than that of wild type in all three time points, indicating many inhibited genes expressed after AbrB gene was knocked out. In Table 3, more than a few hundred genes were up-regulated and down-regulated between wild type and $\triangle \mathrm{AbrB}$ strains in all time points, which supported the above conclusion that $\mathrm{AbrB}$ can regulate numerous genes directly and indirectly. The number of expressed genes of DW2 wild type at $14 \mathrm{~h}$ is greater than that at 22 and $25 \mathrm{~h}$. It is shown that more genes are activated in logarithmic growth phase, while quite a few of them are absent or very lowly expressed in stationary and transitional phases. Both upregulated and down-regulated genes in $\triangle \mathrm{AbrB}$ are far fewer than that of wild type, as shown in Table 3 , may be caused by the inhabitation absence of AbrB. Many regulated genes expressed at all time points after AbrB was knocked out, and no longer were differentially genes. We also note that the

TABLE 2 | General genome features of the four B. licheniformis strains.

\begin{tabular}{|c|c|c|c|c|c|c|c|}
\hline Strain & Size (Mp) & GC $\%$ & No. CDS & No. rRNA & No. tRNA & No. Insertion sequence & No. Tandem repeats \\
\hline B. licheniformis DW2 & 4.47 & 45.93 & 4,717 & 24 & 81 & 71 & 144 \\
\hline B. licheniformis ATCC14580 & 4.22 & 46.19 & 4,173 & 21 & 72 & 57 & 116 \\
\hline B. licheniformis $9945 \mathrm{~A}$ & 4.38 & 45.92 & 4,225 & 21 & 72 & 67 & 116 \\
\hline B. licheniformis WX-02 & 4.29 & 46.06 & 4,512 & 24 & 79 & 62 & 103 \\
\hline
\end{tabular}

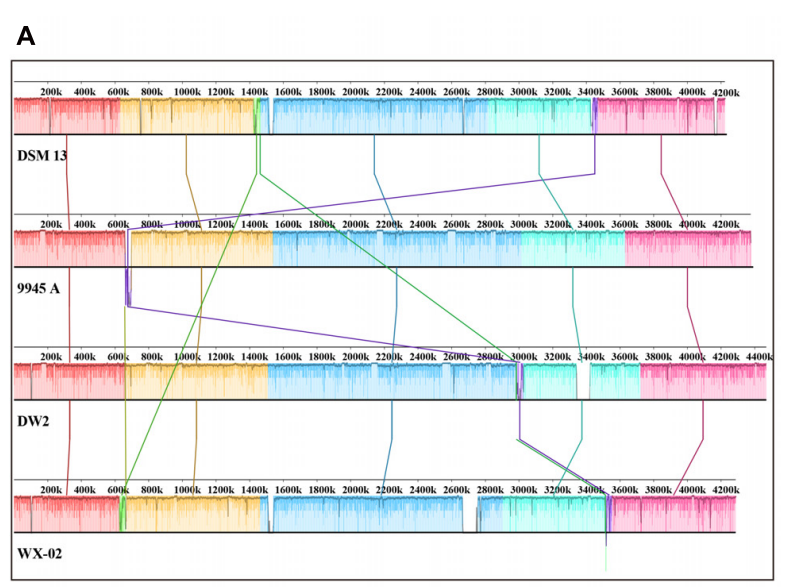

B

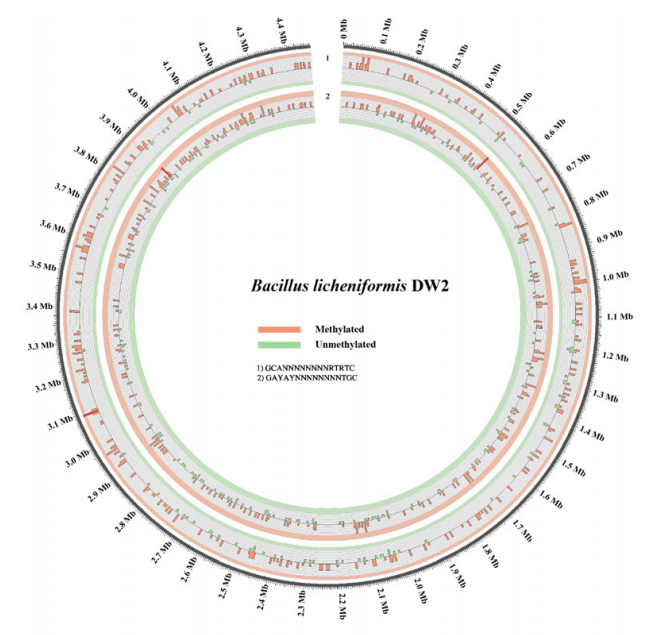

C

\section{。}

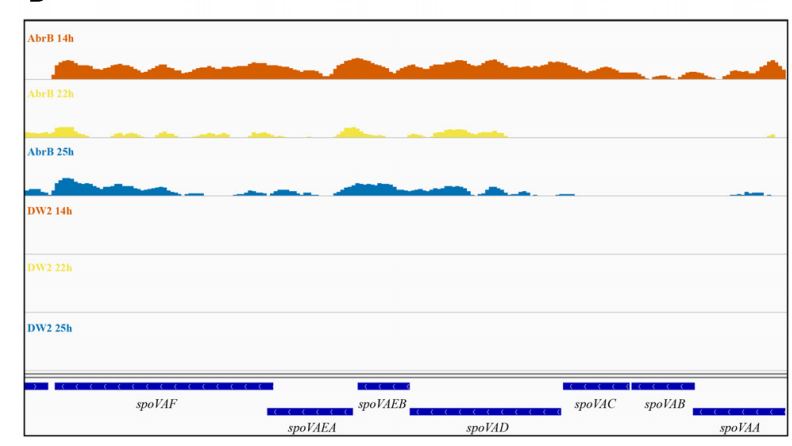

FIGURE 2 | Comparative genomics of four B. licheniformis strains and other genomic features of B. licheniformis DW2. (A) Multiple genomic alignments of four B. licheniformis strains. (B) Distribution of $6 \mathrm{~mA}$ motifs in B. licheniformis DW2 genome. (C) The operon acoABCL associated with acetoin catabolism in B. licheniformis DW2. (D) The stage V sporulation protein in B. licheniformis DW2. 


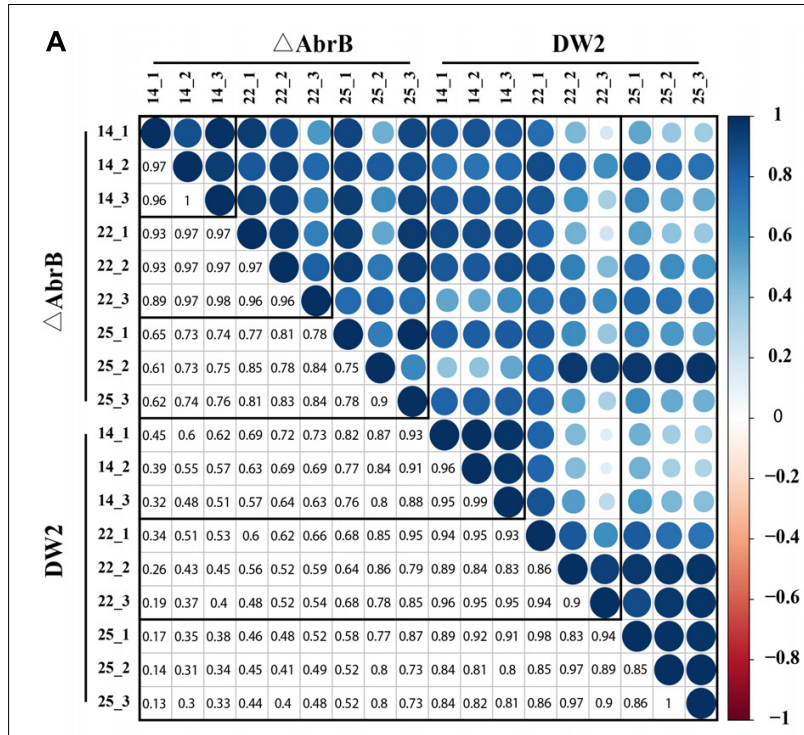

B

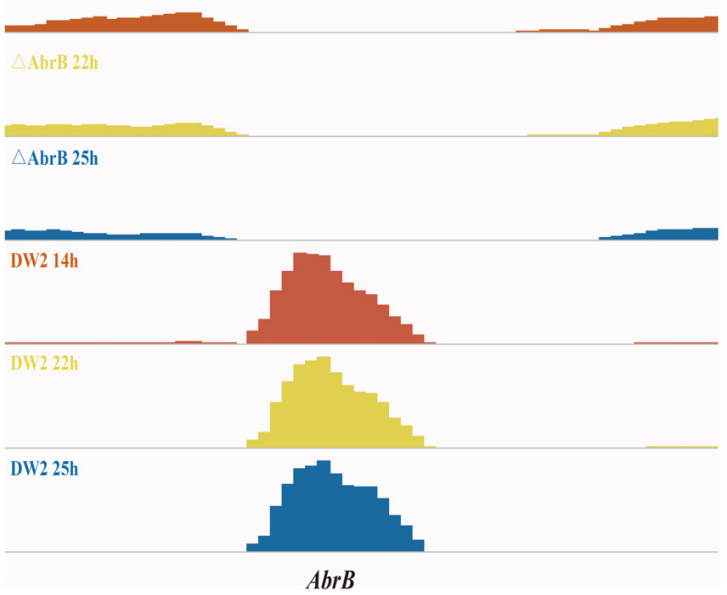

C

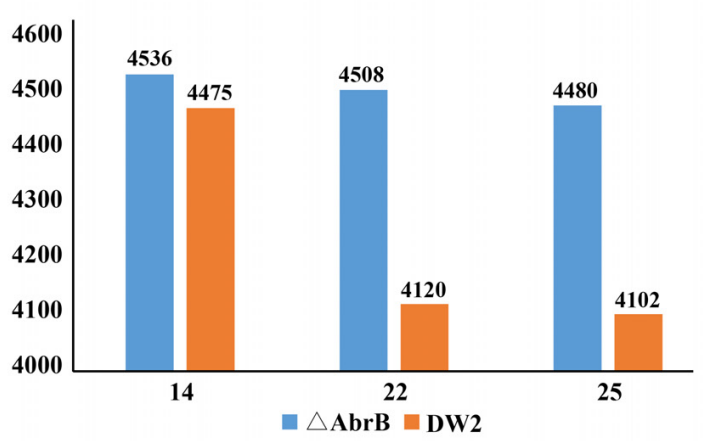

D

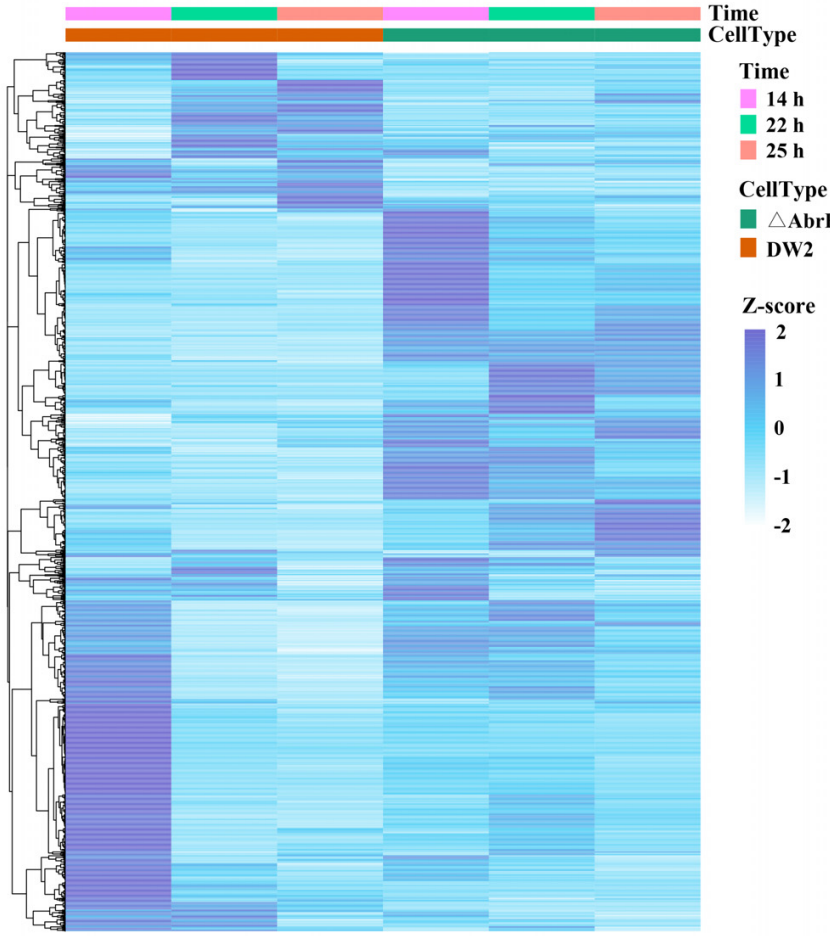

FIGURE 3 | Gene expression of wild B. licheniformis DW2 and AbrB-knockout strains. (A) The correlation of three biological replicates in three time points of wild and AbrB-knockout strains. (B) The AbrB gene was knocked out and not expressed in AbrB-knockout strain. (C) The number of expressed genes in three different time points of wild and AbrB-knockout strains. (D) The different gene expression pattern between wild and AbrB-knockout strains.

number of DEGs correlate with time length. Among three columns either in wild type or $\triangle \mathrm{AbrB}$ type, the middle column represents the DEGs numbers between 25 and $14 \mathrm{~h}$, which are significantly greater the others. On the contrary, the DEGs between 22 and $25 \mathrm{~h}$ with the shortest time length correspond to the smallest numbers. It suggested that more and more genes begin to transcript or stop transcription and become DEGs with the development process of organism. To gain insight into the differential expression of these DEGs, complete linkage hierarchical cluster analysis based on RPKM values was performed using R. The heat map showed significant differential expression between strains, we identified six broad clusters of genes that exhibited expression changes over time. Genes in cluster found that about 30\% of the DEGs in DW2 strains show high expression at logarithmic growth phase $(14 \mathrm{~h})$ and low expression at transitional phase $(22 \mathrm{~h})$ and stationary phase $(25 \mathrm{~h})$.

To understand the function of these DEGs, they were grouped according to their COG functional categories. As shown in Figure 4A, a majority of the functional categories in B. licheniformis DW2 contained both induce and repressed genes, indicating that this bacterium needs to balance many biological pathways under different conditions. We found that the pathways had a very unequal distribution, and some of them were significantly affected, such as 'Carbohydrate transport and metabolism (G),' 'Amino Acid transport and metabolism (E), 'Cell wall/membrane/envelope biogenesis (M),' 'Energy production and conversion (C)' and 'Lipid transport and metabolism (I).' Notably, almost all DEGs in the functional categories of 'cell motility $(\mathrm{N})^{\prime}$ were down-regulated in logarithmic growth phase 
TABLE 3 | The number of DEGs between wild strain and knockout strain.

\begin{tabular}{|c|c|c|c|c|c|c|c|c|c|}
\hline & \multicolumn{3}{|c|}{$\Delta$ AbrB-DW2 } & \multicolumn{3}{|c|}{$\Delta$ AbrB strain } & \multicolumn{3}{|c|}{ DW2 wild strain } \\
\hline & $14 \mathrm{~h}$ & $22 \mathrm{~h}$ & $25 \mathrm{~h}$ & $14-22 \mathrm{~h}$ & $14-25 \mathrm{~h}$ & $22-25 \mathrm{~h}$ & $14-22 \mathrm{~h}$ & $14-25 \mathrm{~h}$ & $22-25 \mathrm{~h}$ \\
\hline Total & 1517 & 1439 & 1416 & 575 & 950 & 369 & 1651 & 1897 & 710 \\
\hline Up & 808 & 790 & 827 & 311 & 406 & 115 & 870 & 965 & 291 \\
\hline Down & 709 & 649 & 589 & 264 & 544 & 254 & 781 & 932 & 419 \\
\hline
\end{tabular}
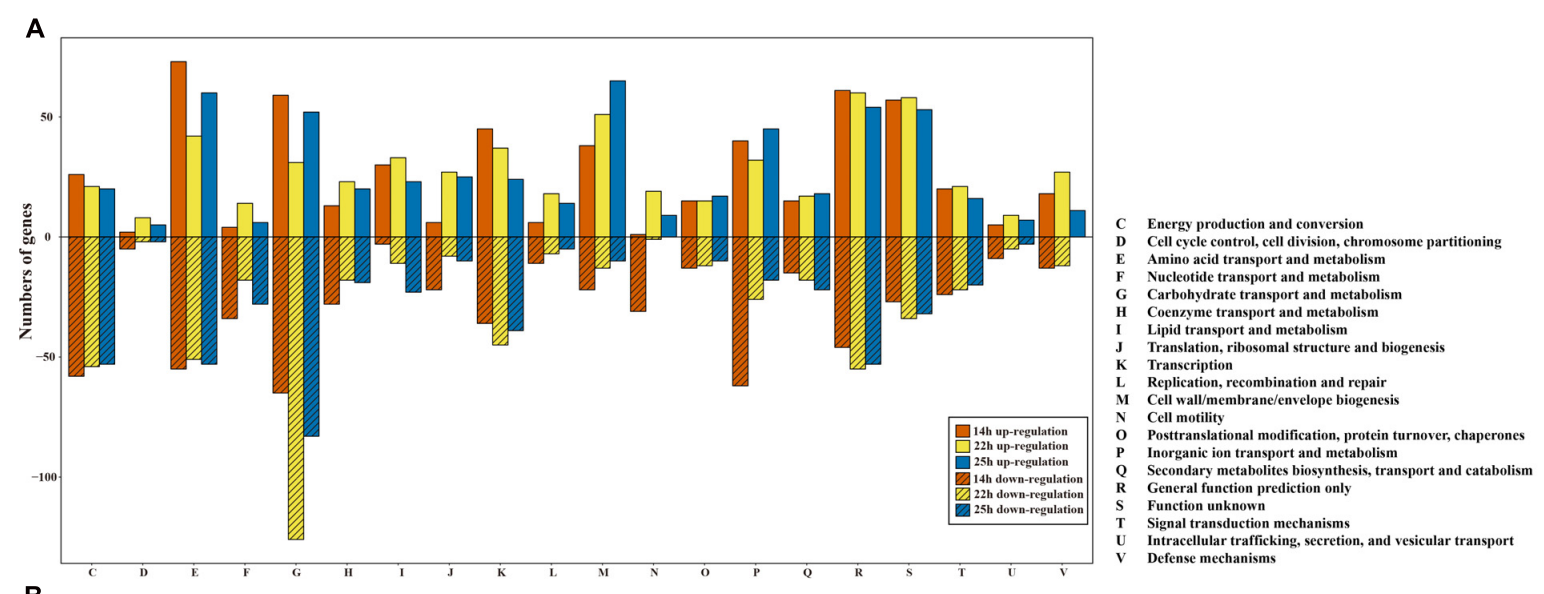

$\mathbf{B}$

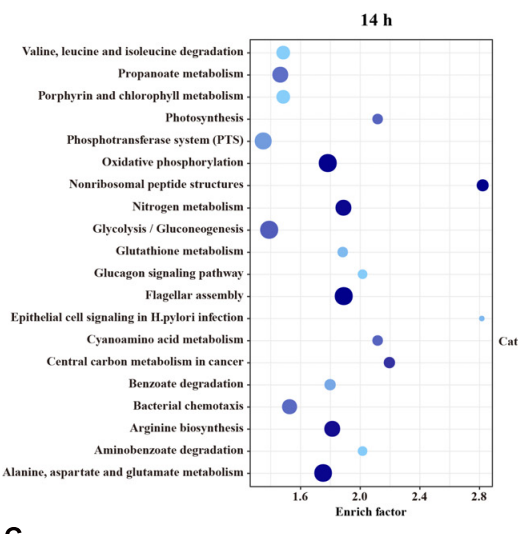

C

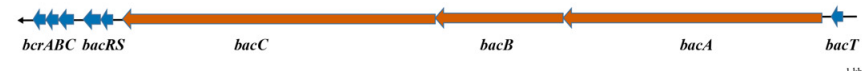

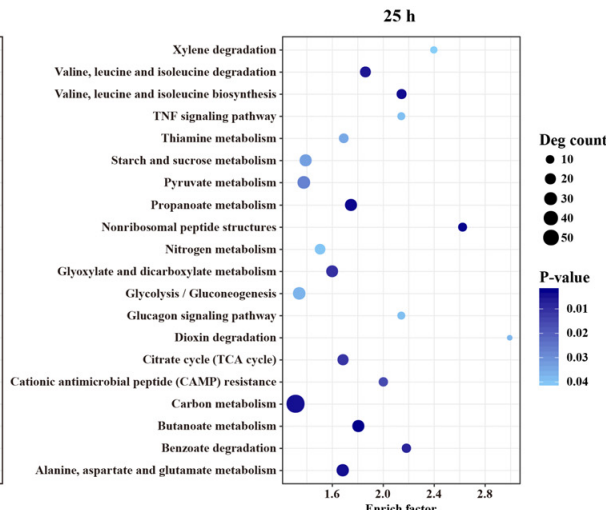

D
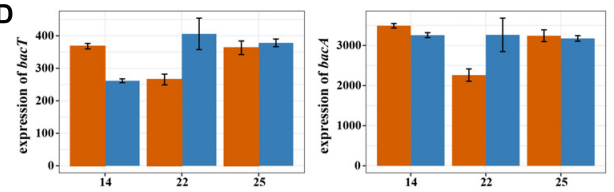

$\triangle{ }_{\mathrm{DW} 2}^{\triangle \mathrm{AbrB}}$

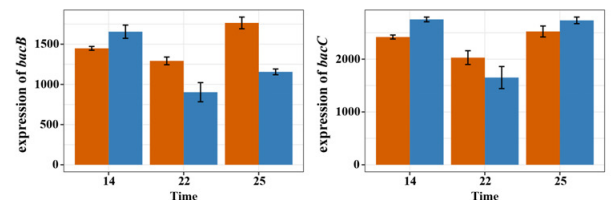

FIGURE 4 | Functional enrichment of DEGs and different expression of bacitracin biosynthesis related genes. (A) The functional categories of DEGs in three different time points of wild type and AbrB-knockout strains. (B) The top 20 significantly enriched pathways of DEGs in three time points. (C) The cluster of bacitracin biosynthesis related genes in B. licheniformis DW2. (D) The different expression of bacitracin biosynthesis related genes bacA, bacB, bacC and bacT between wild and AbrB-knockout strains.

$(14 \mathrm{~h})$ and up-regulated in transitional phase $(22 \mathrm{~h})$ and stationary phase $(25 \mathrm{~h})$. Previous observations suggested one of the major functions of $\mathrm{AbrB}$ in repressing biofilm formation by repressing signal peptidase sip $W$, and sip $W$-processed protein may have a role in a motility structure in B. subtilis (Hamon et al., 2004). We speculated that AbrB repressed some genes related 
to cell motility in transitional phase $(22 \mathrm{~h})$ and stationary phase (25 h).

In addition, we mapped all DEGs to KEGG terms to identify significantly enriched metabolic pathways and got 190 pathways, which contained 1,271 DEGs. To obtain a better visual display of the results, top 20 significantly enriched pathways of each time points were shown in Figure 4B. The pathway flagellar assembly was significantly at logarithmic growth phase $(14 \mathrm{~h})$, which was consistent with the results of COG function enrichment. We also found that the number of genes in significantly enriched pathways was different among three time points, $14 \mathrm{~h}$ is much more than 22 and $25 \mathrm{~h}$. These results also coincide with the variations of DEGs RPKM in $\triangle \mathrm{AbrB}$ strain.

\section{The Expression of Bacitracin Biosynthesis Related Genes}

Bacitracin, comprised of 12 cyclic polypeptides, is an important non-ribosomal peptide antibiotic. It is produced by several Bacillus strains that are usually active against other Gram-positive and some strains of Gram-negative microorganisms, but not against the strain itself. B. subtilis C126 was reported as a bacitracin producer (Azevedo et al., 1993). B. subtilis 168, which has no bacitracin synthetase, but is more sensitive to bacitracin than B. licheniformis, has several homologs of $b c r A B C$ (Ohki et al., 2003). In previous studies, the bacitracin synthetase gene cluster was clearly described (Konz et al., 1997; Neumüller et al., 2001; Mascher et al., 2003). A thioesterase bacT and a nonribosomal peptide synthetase (NPRS) bacABC operon compose the bacitracin synthetase gene cluster in B. licheniformis. The $b c r A B C$ genes of transporter system which are hypothesized to pump out bacitracin from the cells localize downstream of bac $A B C$, between the bacABC operon and transporter system bcr $A B C$ is two-component system bacRS. Using antiSMASH 4.0.0 tool (Blin et al., 2017), we identified a complete bacitracin synthetase gene cluster in B. licheniformis DW2, and all genes of this cluster shows high similarity (identity $>90 \%$ ) to bacitracin synthetase operon in B. licheniformis strain ATCC 10716 (Figure 4C). The bacT gene is 705 bp and encodes a protein of 235 amino acids. The bacABC gene cluster starts from downstream of the $b a c T$ gene and encoding bacitracin synthetase, it consists of the gene $\operatorname{bac} A(15,771 \mathrm{bp}), \operatorname{bacB}(7,809 \mathrm{bp})$, and $\operatorname{bacC}(19,080 \mathrm{bp})$. Gene bacR (717 bp) and bacS (1,035 bp) encode two-component regulatory system bacRS. The bcrABC gene cluster locates after 80 bp downstream of the bacRS genes, which is composed of $b c r A$ (921 bp), $b c r B$ (735 bp), and $\operatorname{bcrC}(612 \mathrm{bp})$ and encoding $\mathrm{ABC}$ transporter. These findings provided gene level information of bacitracin biosynthesis for B. licheniformis DW2.

Previous study suggested that $\mathrm{AbrB}$ might repress the expression of both bacT and bacA through binding to the promoter regions in B. licheniformis (Wang et al., 2017). Although $b a c A B C$ and $b a c T$ are in the same bacitracin synthetase gene cluster, their transcription levels tremendously vary in both wild type and $\triangle \mathrm{AbrB}$ (Figure 4D). The highest expressed gene bacA has about 10-fold RPKM comparing with bacT, the lowest expressed one; while $b a c B$ and $b a c C$ are between the two. In addition, the genes of bacitracin synthetase cluster did not share an identical differentiated expression manner when $\mathrm{AbrB}$ was knocked out, the up/down regulations of bacT and $b a c A B C$ did not show an obvious regularity. However, at $22 \mathrm{~h}$ their expression level of $\triangle \mathrm{AbrB}$ were lower than those of 14 and $25 \mathrm{~h}$, which was found in all the above four genes, especially in bacT and bacA.

Notably, the $b c r A B C$ were significantly up-regulated in $\triangle \mathrm{AbrB}$ strain at all three time points in contrast to wild type (Supplementary Figure S3). In previous study, B. licheniformis was hypothesized to pump out bacitracin for self-resistance by bcrABC (Neumüller et al., 2001). Besides, experiments showed that $\triangle \mathrm{AbrB}$ strains would increase bacitracin yield (Supplementary Figure S2; Wang et al., 2017). Our findings provided evidence for bacitracin up-regulation and transport, which was accordance with the above two studies and linked them together. Comparing with wild type, AbrB-knockout strains secreted more bacitracin, thus they had to increase resistance to the extra bacitracin in order to avoid self-intoxication. The up-regulation of $b c r A B C$ enabled $\triangle A b r B$ strain produce more transporters to remove the bacitracin out of the cell. So it is reasonable to view the differential expression of $\operatorname{bcr} A B C$ as a response to the change of bacitracin production.

\section{CONCLUSION}

In this study, we successfully assemble the complete genome of B. licheniformis DW2 using PacBio SMRT sequencing system and de novo assembly based on the HGAP method. The genomic information obtained in this study would help subsequent comparative genomic analysis with other $B$. licheniformis strains. Meanwhile, we conducted comprehensive analyses of B. licheniformis DW2 by ssRNA-seq in wild and $\triangle \mathrm{AbrB}$ strains. The ssRNA-seq data enabled us to comparatively analyze DEGs between different strains and growth phases and provided us information on bacitracin synthesis. In previous studies, $B$. licheniformis was considered to transport bacitracin for selfresistance by $b c r A B C$ (Neumüller et al., 2001). The global transcription regulator $\mathrm{AbrB}$ represses the transcription of the bacitracin synthase operon by directly binding to the bacitracin synthase operon promoter region (Wang et al., 2017). In our study, we identified a complete bacitracin synthetase gene cluster in the genome including the location and length of each gene. The expressions of $b c r A B C$ were significantly upregulated in $\triangle \mathrm{AbrB}$ strain. These findings provided evidence for bacitracin up-regulation and removing out of the cell, which were consistent with the above two studies and linked them together. This study would be helpful for improving the industrial process of bacitracin production through AbrB-knockout and further understanding of bacitracin transport in B. licheniformis.

\section{ACCESSION NUMBERS}

The genomic and transcriptomic sequencing data of B. licheniformis DW2 generated in this study have been submitted to BIG Data Center (http://bigd.big.ac.cn) under accession numbers GWHAAAE00000000 and CRA000557. 


\section{AUTHOR CONTRIBUTIONS}

L-LC and J-XG conceived the study. C-CS, JG, and J-MS analyzed the data. S-WC and DW performed the experiments. C-CS, L-LC, and J-XG wrote the paper.

\section{FUNDING}

This work was supported by National Natural Science Foundation of China $(31271406,31571351)$ and the Natural Science Foundation of Hubei Province (2015CFA044).

\section{REFERENCES}

Ali, N. O., Bignon, J., Rapoport, G., and Debarbouille, M. (2001). Regulation of the acetoin catabolic pathway is controlled by sigma $\mathrm{L}$ in Bacillus subtilis. J. Bacteriol. 183, 2497-2504. doi: 10.1128/JB.183.8.2497-2504.2001

Anders, S., and Huber, W. (2010). Differential expression analysis for sequence count data. Genome Biol. 11:R106. doi: 10.1186/gb-2010-11-10-r106

Aziz, R. K., and Bartels, D., Best, A. A., DeJongh, M., Disz, T., and Edwards, R. A. (2008). The RAST server: rapid annotations using subsystems technology. BMC Genomics 9:75. doi: 10.1186/1471-2164-9-75

Azevedo, E. C., Rios, E. M., Fukushima, K., and Campos-Takaki, G. M. (1993). Bacitracin production by a new strain of bacillus subtilis. Appl. Biochem. Biotechnol. 42, 1-7. doi: 10.1007/BF02788897

Banse, A. V., Chastanet, A., Rahn-Lee, L., Hobbs, E. C., and Losick, R. (2008). Parallel pathways of repression and antirepression governing the transition to stationary phase in Bacillus subtilis. Proc. Natl. Acad. Sci. U.S.A. 105, 15547-15552. doi: 10.1073/pnas.0805203105

Benson, G. (1999). Tandem repeats finder: a program to analyze DNA sequences. Nucleic Acids Res. 27, 573-580. doi: 10.1093/nar/27.2.573

Blin, K., Wolf, T., Chevrette, M. G., Lu, X., Schwalen, C. J., Kautsar, S. A., et al. (2017). antiSMASH 4.0-improvements in chemistry prediction and gene cluster boundary identification. Nucleic Acids Res. 45, W36-W41. doi: 10.1093/nar/ gkx319

Cain, B. D., Norton, P. J., Eubanks, W., and Nick, H. S. Allen, C. M. (1993). Amplification of the bacA gene confers bacitracin resistance to Escherichia coli. J. Bacteriol. 175, 3784-3789. doi: 10.1128/jb.175.12.3784-3789.1993

Chalker, A. F., Ingraham, K. A., Lunsford, R. D., Bryant, A. P., Bryant, J., Wallis, N. G., et al. (2000). The bacA gene, which determines bacitracin susceptibility in Streptococcus pneumoniae and Staphylococcus aureus, is also required for virulence. Microbiology 146, 1547-1553. doi: 10.1099/00221287-146-7- 1547

Chin, C. S., Alexander, D. H., Marks, P., Klammer, A. A., Drake, J., Heiner, C., et al. (2013). Nonhybrid, finished microbial genome assemblies from long-read SMRT sequencing data. Nat. Methods 10, 563-569. doi: 10.1038/nmeth.2474

Chumsakul, O., Takahashi, H., Oshima, T., Hishimoto, T., Kanaya, S., Ogasawara, N., et al. (2011). Genome-wide binding profiles of the Bacillus subtilis transition state regulator $\mathrm{AbrB}$ and its homolog $\mathrm{Abh}$ reveals their interactive role in transcriptional regulation. Nucleic Acids Res. 39, 414-428. doi: 10.1093/nar/gkq780

Darling, A. E., Mau, B., and Perna, N. T. (2010). Progressive Mauve: multiple genome alignment with gene gain, loss and rearrangement. PLoS One 5:e11147. doi: 10.1371/journal.pone.0011147

El-Sharnouby, S., Redhouse, J., and White, R. A. (2013). Genome-wide and cellspecific epigenetic analysis challenges the role of polycomb in Drosophila spermatogenesis. PLoS Genet. 9:e1003842. doi: 10.1371/journal.pgen.1003842

Flusberg, B. A., Webster, D. R., Lee, J. H., Travers, K. J., Olivares, E. C., Clark, T. A., et al. (2010). Direct detection of DNA methylation during single-molecule, real-time sequencing. Nat. Methods 7, 461-465. doi: 10.1038/nmeth.1459

Galperin, M. Y., Makarova, K. S., Wolf, Y. I., and Koonin, E. V. (2015). Expanded microbial genome coverage and improved protein family annotation in the COG database. Nucleic Acids Res. 43, 261-269. doi: 10.1093/nar/gku1223

Gene Ontology Consortium (2015). Gene ontology consortium: going forward. Nucleic Acids Res. 43, D1049-1056. doi: 10.1093/nar/gku1179

\section{ACKNOWLEDGMENTS}

The authors are grateful to Dr. Jack Chen at Simon Fraser University for valuable suggestions in data analysis.

\section{SUPPLEMENTARY MATERIAL}

The Supplementary Material for this article can be found online at: https://www.frontiersin.org/articles/10.3389/fmicb. 2018.00307/full\#supplementary-material

Guo, J., Cheng, G., Gou, X. Y., Xing F., Li S., Han, Y. C., et al. (2015) Comprehensive transcriptome and improved genome annotation of Bacillus licheniformis WX-02. FEBS Lett. 589, 2372-2381. doi: 10.1016/j.febslet.2015. 07.029

Hamon, M. A., Stanley, N. R., Britton, R. A., Grossman, A. D., and Lazazzera, B. A. (2004). Identification of AbrB-regulated genes involved in biofilm formation by Bacillus subtilis. Mol. Microbiol. 52, 847-860. doi: 10.1111/j.1365-2958.2004. 04023.x

Johnson, B. A., and Anker, H. Meleney, F. L. (1945). Bacitracin: a new antibiotic produced by a member of the B. subtilis group. Science 102, 376-377. doi: 10.1126/science.102.2650.376

Kim, H. J., Kim, S. I., Ratnayake-lecamwasam, M., Tachikawa, K., Sonenshein, A. L., and Strauch, M. (2003). Complex regulation of the Bacillus subtilis aconitase gene. J. Bacteriol. 185, 1672-1680. doi: 10.1128/JB.185.5.1672-1680. 2003

Konglom, N., Chuensangjun, C., Pechyen, C., and Sirisansaneeyakul, S. (2012). Production of poly- $\gamma$-glutamic acid by Bacillus licheniformis, synthesis and characterization. J. Met. Mater. Miner. 22, 7-11.

Konz, D., Klens, A., Schörgendorfer, K., and Marahiel, M. A. (1997). The bacitracin biosynthesis operon of Bacillus licheniformis ATCC 10716: molecular characterization of three multi-modular peptide synthetases. Chem. Biol. 4, 927-937. doi: 10.1016/S1074-5521(97)90301-X

Kumar, S., Cheng, X., Klimasauskas, S., Mi, S., Posfai, J., Roberts, R. J., et al. (1994). The DNA (cytosine-5) methyltransferases. Nucleic Acids Res. 22, 1-10. doi: $10.1093 /$ nar/22.1.1

Kunst, F., Ogasawara, N., Moszer, I., Albertini, A. M., Alloni, G., Azevedo, V., et al. (1997). The complete genome sequence of the gram-positive bacterium Bacillus subtilis. Nature 390, 249-256. doi: 10.1038/36786

Kurtz, S., Phillippy, A., Delcher, A. L., Smoot, M., Shumway, M., Antonescu, C., et al. (2004). Versatile and open software for comparing large genomes. Genome Biol. 5:R12. doi: 10.1186/gb-2004-5-2-r12

Lagesen, K., Hallin, P., Rødland, E. A., Staerfeldt, H. H., Rognes, T., and Ussery, D. W. (2007). RNAmmer: consistent and rapid annotation of ribosomal RNA genes. Nucleic Acids Res. 35, 3100-3108. doi: 10.1093/nar/ gkm160

Langmead, B., and Salzberg, S. L. (2012). Fast gapped-read alignment with bowtie 2. Nat. Methods 9, 357-359. doi: 10.1038/nmeth.1923

Laird, P. W. (2010). Principles and challenges of genome-wide DNA methylation analysis. Nat. Rev. Genet. 11, 191-203. doi: 10.1038/nrg2732

Li, E., Yang, H., Wang, X., Wan, L. Pan, H., and Zhu, D. (2015). Whole-genome sequencing and analysis of inosine-producing strain Bacillus subtilis ATCC 13952. Acta Microbiol. Sin. 55, 1560-1567.

Liu, H., Yin, S., An, L., Zhang, G., Cheng, H., Xi, Y., et al. (2016). Complete genome sequence of Bacillus subtilis BSD-2, a microbial germicide isolated from cultivated cotton. J. Biotechnol. 230, 26-27. doi: 10.1016/j.jbiotec.2016. 05.019

Liu, Y., Zhang, S., Yong, Y.-C., Ji, Z., Ma, X., Xu, Z., et al. (2011). Efficient production of acetoin by the newly isolated Bacillus licheniformis strain MEL09. Process Biochem. 46, 390-394. doi: 10.1016/j.procbio.2010.07.024

Lowe, T. M., and Eddy, S. R. (1997). tRNAscan-SE: a program for improved detection of transfer RNA genes in genomic sequence. Nucleic Acids Res. 25, 955-964. doi: 10.1093/nar/25.5.0955 
Mao, X., Ma, Q., Zhou, C., Chen, X., Zhang, H., Yang, J., et al. (2014). Door 2.0: presenting operons and their functions through dynamic and integrated views. Nucleic Acids Res. 42, 654-659. doi: 10.1093/nar/gkt 1048

Mascher, T., Margulis, N. G., Wang, T., Ye, R. W., and Helmann, J. D. (2003). Cell wall stress responses in Bacillus subtilis: the regulatory network of the bacitracin stimulon. Mol. Microbiol. 50, 1591-1604. doi: 10.1046/j.1365-2958.2003. 03786.x

Mcinerney, M. J., Javaheri, M., and Nagle, D. P. (1990). Properties of the biosurfactant produced by bacillus licheniformis strain JF-2. J. Ind. Microbiol. 5, 95-101. doi: 10.1007/BF01573858

Mortazavi, A., Williams, B. A., McCue, K., Schaeffer, L., and Wold, B. (2008). Mapping and quantifying mammalian transcriptomes by RNA-Seq. Nat. Methods 5, 621-628. doi: 10.1038/nmeth. 1226

Neumüller, A. M., Konz, D., and Marahiel, M. A. (2001). The two-component regulatory system BacRS is associated with bacitracin 'self-resistance' of Bacillus licheniformis ATCC 10716. Eur. J. Biochem. 268, 3180-3189. doi: 10.1046/j. 1432-1327.2001.02203.x

Ogata, H., Goto, S., Sato, K., Fujibuchi, W., Bono, H., and Kanehisa, M. (1999). KEGG: kyoto encyclopedia of genes and genomes. Nucleic Acids Res. 27, 29-34. doi: 10.1093/nar/27.1.29

Ohki, R., Giyanto, Tateno, K., Masuyama, W., Moriya, S., and Kobayashi, K. (2003). The BceRS two-component regulatory system induces expression of the bacitracin transporter, BceAB, in Bacillus subtilis. Mol. Microbiol. 49, 1135-1144. doi: 10.1046/j.1365-2958.2003.03653.x

Perez-Valdespino, A., Li, Y., Setlow, B., Ghosh, S., Pan, D., and Korza, G. (2014). Function of the SpoVAEa and SpoVAF proteins of Bacillus subtilis spores. J. Bacteriol. 196, 2077-2088. doi: 10.1128/JB. 01546-14

Podlesek, Z., Comino, A., Herzog-Velikonja, B., Zgur-Bertok, D., Komel, R., and Grabnar, M. (1995). Bacillus licheniformis bacitracin resistance $\mathrm{ABC}$ transporter: relationship to mammalian multidrug resistance. Mol. Microbiol. 16, 969-976. doi: 10.1111/j.1365-2958.1995.tb0 2322.x

Podlesek, Z., Comino, A., Herzog-Velikonja, B., and Grabnar, M. (2000). The role of the bacitracin $\mathrm{ABC}$ transporter in bacitracin resistance and collateral detergent sensitivity. FEMS Microbiol. Lett. 188, 103-106. doi: 10.1111/j.15746968.2000.tb09176.x

Pollock, T. J., Thorne, L., Yamazaki, M., and Mikolajczak, M. J., and Armentrout, R. W. (1994). Mechanism of bacitracin resistance in Gram-negative bacteria that synthesize exopolysaccharides. J. Bacteriol. 176, 6229-6237. doi: 10.1128/ jb.176.20.6229-6237.1994

Pötter, M., Oppermann-Sanio, F. B., and Steinbüchel, A. (2001). Cultivation of bacteria producing polyamino acids with liquid manure as carbon and nitrogen source. Appl. Environ. Microbiol. 67, 617-622. doi: 10.1128/AEM.67.2.617-622. 2001

Qian, Q., Lee, C. Y., Helmann, J. D., and Strauch, M. A. (2002). AbrB is a regulator of the sigma(w) regulon in Bacillus subtilis. FEMS Microbiol. Lett. 211, 219-223.
Quinlan, A. R., and Hall, I. M. (2010). BEDTools: a flexible suite of utilities for comparing genomic features. Bioinformatics 26, 841-842. doi: 10.1093/ bioinformatics/btq033

Sitaraman, R. (2016). The role of DNA restriction-modification systems in the biology of Bacillus anthracis. Front. Microbiol. 7, 1-6. doi: 10.3389/fmicb.2016. 00011

Roberts, R. J., Vincze, T., Posfai, J., and Macelis, D. (2015). Rebase-a database for DNA restriction and modification: enzymes, genes and genomes. Nucleic Acids Res. 43, D298-299. doi: 10.1093/nar/gku1046

Sambrook, J., Fritsch, E. F., and Maniatis, T. (1982). Molecular Cloning: A Laboratory Manual. Cold Spring Harbor, NY: Cold Spring Harbor laboratory press.

Sánchezromero, M. A., Cota I., and Casadesús, J. (2015). DNA methylation in bacteria: from the methyl group to the methylome. Curr. Opin. Microbiol. 25, 9-16. doi: 10.1016/j.mib.2015.03.004

Shafikhani, S. H., and Leighton, T. (2004). AbrB and Spo0E control the proper timing of sporulation in Bacillus subtilis. Curr. Microbiol. 48, 262-269. doi: 10.1007/s00284-003-4186-2

Sommer, D. D., Delcher, A. L., Salzberg, S. L., and Pop, M. (2007). Minimus: a fast, lightweight genome assembler. BMC Bioinformatics 8:64. doi: 10.1186/14712105-8-64

Strauch, M., Webb, V., Spiegelman, G., and Hoch, J. A. (1990). The SpoOA protein of Bacillus subtilis is a repressor of the abrB gene. Proc. Natl. Acad. Sci. U.S.A. 87, 1801-1805. doi: 10.1073/pnas.87.5.1801

Strauch, M. A., Bobay, B. G., Cavanagh, J., Yao, F., Wilson, A., and Le, B. Y. (2007). Abh and AbrB control of Bacillus subtilis antimicrobial gene expression. J. Bacteriol. 189, 7720-7732. doi: 10.1128/JB.01081-07

Veith, B., Herzberg, C., Steckel, S., Feesche, J., Maurer, K. H., Ehrenreich, P., et al. (2004). The complete genome sequence of Bacillus licheniformis DSM13, an organism with great industrial potential. J. Mol. Microbiol. Biotechnol. 7, 204-211. doi: 10.1159/000079829

Wang, D., Wang, Q., Qiu, Y., Nomura, C. T., Li, J., and Chen, S. (2017). Untangling the transcription regulatory network of the bacitracin synthase operon in Bacillus licheniformis DW2. Res. Microbiol. 168, 515-523. doi: 10.1016/j.resmic. 2017.02.010

Zhang, Z., Schwartz, S., Wagner, L., and Miller, W. (2000). A greedy algorithm for aligning DNA sequences. J. Comput. Biol. 7, 203-214. doi: 10.1089/ 10665270050081478

Conflict of Interest Statement: The authors declare that the research was conducted in the absence of any commercial or financial relationships that could be construed as a potential conflict of interest.

Copyright $\odot 2018$ Shu, Wang, Guo, Song, Chen, Chen and Gao. This is an openaccess article distributed under the terms of the Creative Commons Attribution License (CC BY). The use, distribution or reproduction in other forums is permitted, provided the original author(s) and the copyright owner are credited and that the original publication in this journal is cited, in accordance with accepted academic practice. No use, distribution or reproduction is permitted which does not comply with these terms. 